\title{
Genetic characterization of Bacillus anthracis in Guizhou Province, Southwest of China
}

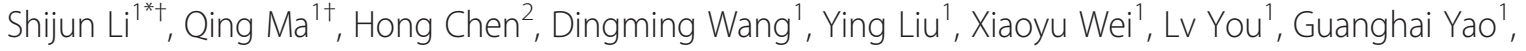 \\ Kecheng $\operatorname{Tian}^{1}$ and Guangpeng Tang ${ }^{1}$
}

\begin{abstract}
Background: Bacillus (B.) anthracis is the pathogen that causes fatal anthrax. Guizhou Province is an old foci of anthrax in the southwest of China. Human anthrax has also been frequently reported in Guizhou in recent year. However, there is limited information on the genetic background of local B. anthracis isolates in Guizhou Province. Strain-specific detection of this bacterium using molecular approaches has enhanced our knowledge of microbial genetics. In the present study, we employed Multiple Locus Variable Number Tandem Repeats (VNTR) Analysis (MLVA) assay to analyze the genetic characteristics of $B$. anthracis strains isolated in Guizhou Province and their relationships to worldwide distributed isolates.
\end{abstract}

Results: A total of 32 isolates of B. anthracis from soil, human, cattle, dog and water of different anthrax epidemics in Guizhou Province from 2006 to 2011 were confirmed with phage lysis test, penicillin inhibition test and PCR. MLVA-8 discriminated them into 28 unique MLVA types (MT G1 - G28), which were novel MTs compared with the previous reports. Cluster tree based on 32 isolates from Guizhou Province and 76 worldwide distributed isolates (30 MTs) showed they were divided into three clusters, designated A, B and C. All the 32 isolates were distributed in cluster $A$, which were further grouped into $A 1, A 2, A 3$ and $A 4$ sub-clusters.

32 isolates from Guizhou Province were closely grouped in each of the sub-clusters, respectively. Minimum Spanning Tree (MST) based on the MLVA data showed that the 28 MLVA profiles of isolates from Guizhou Province and 30 MLVA profiles of worldwide distributed isolates formed three clonal complexes (CCs) and ten singletons.

Conclusions: 28 novel MTs of B. anthracis from Guizhou were revealed and their relationships to worldwide isolates were showed. The results will provide important information for prevention of anthrax and also enhances our understanding of genetic characteristics of $B$. anthracis in China.

Keywords: Anthrax, Bacillus anthracis, MLVA, Genetic characterization

\section{Background}

Anthrax is an often fatal bacterial infection that occurs when Bacillus anthracis endospores enter the body through abrasions in the skin or by inhalation or ingestion $[1,2]$. It is a zoonosis to which most mammals, especially grazing herbivores, are considered susceptible [2]. While anthrax currently affects mostly livestock and wildlife around the world, it can and does kill humans [3]. Humans almost invariably contract anthrax from

\footnotetext{
*Correspondence: zjumedjun@163.com

${ }^{\dagger}$ Equal contributors

'Institute of communicable disease control and prevention, Guizhou Provincial Centre for Disease Control and Prevention, 101 Bageyan Road, Guiyang 550004, Guizhou, People's Republic of China

Full list of author information is available at the end of the article
}

handling infected animals or carcasses of animals that have died of the disease, or meat, skins, hair, bones, etc. from such animals [4]. Anthrax infection in humans occurs by three major routes, the skin, the respiratory tract or the gastro-intestinal tract, generating three different primary forms of the disease, the cutaneous, the inhalational and the gastro-intestinal forms [2]. Cutaneous anthrax, the most common form, occurs as a result of contamination of skin with the bacterial spores, due to its mechanical abrasion or damage caused by insect bites [5]. Besides, the great current interest in anthrax is due to its potential as a bioterrorism and biowarfare agent $[6,7]$.

Ancient Chinese medical books suggest that an anthraxlike disease has been present in China for more than 
5,000 years and the epidemiology and symptoms of anthrax had been described [8]. An anthrax surveillance and control project reported 72 outbreaks and 8,988 human cases in ten provinces (Sichuan, Tibet, Inner Mongolia, Xinjiang, Qinghai, Gansu, Guangxi, Guizhou, Yunnan and Hunan) in China in 1990-1994, which indicates a long history of anthrax epidemics in China [8]. More recent reports showed the sporadic epidemics or outbreaks of anthrax in many provinces of China, such as, Mongolia, Liaoning and Jiangsu [9-11].

Guizhou Province, with nearly 50 million people, is an old foci of anthrax in the southwest of China. For example, a total of 17,975 cases of anthrax in Guizhou Province from 1957 to 1999 had been reported, and the epidemics covered all of the nine prefectures, including Guiyang, Qianxinan, Qiandongnan, Qiannan, Anshun, Bijie, Liupanshui, Tongren and Zunyi, in Guizhou Province [12]. Human anthrax has also been frequently reported in Guizhou in recent year. For instance, there were 32 outbreaks of anthrax occurred in Guzhou Province [13]. An more recent outbreak of cutaneous anthrax including seven cases has been reported in a village of Wangmo County in Guizhou Province in 2010 [14]. However, there is limited information on the genetic background of local B. anthracis isolates in Guizhou Province. In this study, we applied MLVA- 8 to analyze the genetic characteristics of $B$. anthracis strains isolated from Guizhou Province.

\section{Methods}

\section{Bacterial isolates and confirmation}

A total of 32 of isolates were used for analysis in this study (Table 1). All of these isolates were come from the local epidemics of cutaneous anthrax in Guizhou Province from 2006-2011, which included isolates from human and epidemiologic related animal (dog and cattle), soil and water in each epidemic. The localities covered Prefecture Qianxinan, Qiannan, Anshun, Tongren, and Bijie. All the isolates used in this study were confirmed with PCR kit (Takara, Janpan) and conventional methods including phage lysis test, penicillin inhibition test for anthrax diagnosis.

\section{DNA templates preparation}

DNA templates for PCR were prepared directly from bacterial colonies by the boiling method [3]. Briefly, $B$. anthracis cells were streaked onto blood agar plates and then incubated at $37^{\circ} \mathrm{C}$ overnight. A single colony from each plate was transferred into a microcentrifuge tube containing $100 \mu \mathrm{l}$ of DNA extraction liquid (Liferiver, China). The colony was resuspended by vortexing or repetitive pipetting. The cellular suspension was heated to $95^{\circ} \mathrm{C}$ for $20 \mathrm{~min}$ and then cooled to room temperature.
Cellular debris was removed by centrifugation at $15,000 \mathrm{~g}$ for $5 \mathrm{~min}$. The supernatant, containing DNA, was used as the template for PCR amplification.

\section{MLVA experiments}

For the MLVA test, we utilized primers that flank the eight VNTR regions (vrrA, vrrB1, vrrB2, vrrC1, vrrC2, CG3, pXO1, pXO2) as described by previous study $[3,15]$. Amplifications were performed in $50 \mu \mathrm{l}$ total volumes of PCR reaction system contained approximately $25 \mu \mathrm{l}$ of PreMix Taq (TaKaRa, Otsu, Japan), $2 \mu \mathrm{l}$ of forward and reverse primers with concentrations of $10 \mathrm{pmol} / \mu \mathrm{l}, 2 \mu \mathrm{l}$ of DNA, $19 \mu \mathrm{l}$ of deionized water, respectively. Amplification was performed on an Biometra TProfession thermocycler (Biometra, Goettingen, Germany) using amplification parameters included an initial denaturation at $94^{\circ} \mathrm{C}$ for 5 minutes, followed by 34 cycles of $94^{\circ} \mathrm{C}$ for 20 seconds, $60^{\circ} \mathrm{C}$ for 20 seconds, $65^{\circ} \mathrm{C}$ for 20 seconds, then $65^{\circ} \mathrm{C}$ for 5 minutes. PCR products were detected by electrophoresis of $1 \mu \mathrm{l}$ of each reaction on a $1.2 \%$ agarose gel for $30 \mathrm{~min}$ at $100 \mathrm{~V}$, and were sequenced by ABI PRISM 377 DNA sequencer. The full length of each locus were obtained by assembling of forward and reverse sequences using ContigExpress (Invitrogen Life Science, Carlsbad, $\mathrm{CA}$ ), on which the forward sequence and the reverse complement sequence of reverse primer were marked to determine the actual length of amplification. The actual length were calibrated to the nearest sizes for the corresponding VNTR in the report of Keim et al. [3].

\section{Data analysis}

Each unique profile based on allelic combination was designated a unique MLVA type (MT). The Bionumerics software package, version 4.0 (Applied Maths, Belgium) was used for UPGMA clustering analysis based on categorical coefficient, and minimum spanning tree algorithm was used to construct a minimum spanning tree (MST) to determine phylogenetic pattern. The MLVA data of isolates of other provinces in China and worldwide isolates were come from B. anthracis data base (http://mlva.u-psud.fr/ MLVAnet/spip.php?article123).

\section{Ethic statements and biosafety containment}

All experiments involving $B$. anthracis including isolates from human, animal and environments were performed according to the General Requirements for Bio-safety (GB 19489-2008) and approved by the Biosafety Committee of Guizhou Provincial Centre for Disease Control and Prevention, and were also approved by the Ethics Review Committee of Guizhou Provincial Centre for Disease Control and Prevention. 
Table 1 Backgroud information and pathogenic plasmid content of B. anthracis isolates used in this study

\begin{tabular}{|c|c|c|c|c|c|c|c|}
\hline Strains & Original no. & Source & Prefecture & County & Year & pX01 & $\mathrm{pXO2}$ \\
\hline GZBA01 & 2006001 & Dog & Qianxinan & Ceheng & 2006 & + & + \\
\hline GZBA02 & 2006002 & Dog & Qianxinan & Ceheng & 2006 & + & + \\
\hline GZBA03 & 2006003 & Soil & Qianxinan & Zhenfeng & 2006 & + & + \\
\hline GZBA04 & 2006004 & Human & Qianxinan & Zhenfeng & 2006 & + & + \\
\hline GZBA05 & 2006005 & Soil & Qianxinan & Zhenfeng & 2006 & + & + \\
\hline GZBA06 & 2006006 & Soil & Qianxinan & Zhenfeng & 2006 & + & + \\
\hline GZBA07 & 2007001 & Soil & Qianxinan & Ceheng & 2007 & + & + \\
\hline GZBA08 & 2007003 & Soil & Anshun & Zhenning & 2007 & + & + \\
\hline GZBA09 & 2007004 & Soil & Qiannan & Luodian & 2007 & + & + \\
\hline GZBA10 & 2007005 & Soil & Anshun & Zhenning & 2007 & + & + \\
\hline GZBA11 & 2007007 & Water & Qianxinan & Ceheng & 2007 & + & + \\
\hline GZBA12 & 2007008 & Soil & Qianxinan & Ceheng & 2007 & + & + \\
\hline GZBA13 & 2007013 & Soil & Qianxinan & Ceheng & 2007 & + & + \\
\hline GZBA14 & 2007018 & Soil & Qianxinan & Zhenfeng & 2007 & + & + \\
\hline GZBA15 & 2007019 & Soil & Qianxinan & Ceheng & 2007 & + & + \\
\hline GZBA16 & 2007022 & Soil & Qianxinan & Ceheng & 2007 & + & + \\
\hline GZBA17 & 2007023 & Soil & Qiannan & Luodian & 2007 & + & + \\
\hline GZBA18 & 2007024 & Soil & Qianxinan & Ceheng & 2007 & + & + \\
\hline GZBA19 & 2007029 & Soil & Qiannan & Luodian & 2007 & + & + \\
\hline GZBA20 & 2008001 & Water & Qianxinan & Ceheng & 2008 & + & + \\
\hline GZBA21 & 2008002 & Soil & Qiannan & Duyun & 2008 & + & + \\
\hline GZBA22 & 2009001 & Soil & Qiannan & Guiding & 2009 & + & + \\
\hline GZBA23 & 2009002 & Soil & Bijie & Dafang & 2009 & + & + \\
\hline GZBA24 & 2009003 & Soil & Bijie & Zhijin & 2009 & + & + \\
\hline GZBA25 & 2009024 & Soil & Qianxinan & Zhenfeng & 2009 & + & + \\
\hline GZBA26 & 2010001 & Soil & Qianxinan & Anlong & 2010 & + & + \\
\hline GZBA27 & 2010002 & Soil & Qianxinan & Wangmo & 2010 & + & + \\
\hline GZBA28 & 2010007 & Soil & Qianxinan & Wangmo & 2010 & + & + \\
\hline GZBA29 & 2010010 & Cattle & Qianxinan & Wangmo & 2010 & + & + \\
\hline GZBA30 & 2010011 & Soil & Qianxinan & Anlong & 2010 & + & + \\
\hline GZBA31 & 2011002 & Soil & Tongren & Sinan & 2011 & + & + \\
\hline GZBA32 & 2011003 & Soil & Qianxinan & Zhenfeng & 2011 & + & + \\
\hline
\end{tabular}

\section{Results}

\section{Bacteria distribution and confirmation}

All the 32 isolates of $B$. anthracis were confirmed with phage lysis test, penicillin inhibition test and PCR (Table 1). Among these isolates, 26 isolates were isolated from soil, with one from human, one from cattle, two from dog and two from water. According to the year of isolation, six isolates were isolated in 2006, with 13 from 2007, six from 2008, four from 2009, five from 2010 and two from 2011. Based on the origin of region, 21 isolates were from Prefecture Qianxinan with two from Anshun, five from Qiannan, two from Bijie and one from Tongren.

\section{MLVA based genotypes}

MLVA based on eight VNTR loci were performed to characterize the local isolates of $B$. anthracis in Guizhou Province. The copy numbers of each VNTR locus are listed in Table 2. The 32 B. anthracis isolates, based on the unique MLVA profiles, were discriminated into 28 unique MLVA types (MTs), which were nominated as MT G1 - G28 (Figure 1). Among the 28 MTs, MT G1, G5, G13 and G19 included two isolates, respectively, while each of the remaining 24 MTs only contained one isolates. Further, all the MLVA profiles based on eight loci were different from the 30 represent profiles (MT 
Table 2 Observed fragment sizes and copy numbers at eight MLVA loci in the B. anthracis strains isolated in Guizhou Province

\begin{tabular}{|c|c|c|c|c|c|c|c|c|c|c|c|c|c|c|c|c|c|c|}
\hline \multirow[t]{3}{*}{ Strains } & \multirow[t]{3}{*}{ Original no. } & \multirow[t]{3}{*}{ Year } & \multicolumn{16}{|c|}{ MLVA profiles } \\
\hline & & & \multicolumn{2}{|c|}{ vrrA } & \multicolumn{2}{|c|}{ vrrB1 } & \multicolumn{2}{|c|}{ vrrB2 } & \multicolumn{2}{|c|}{ vrrC1 } & \multicolumn{2}{|c|}{ vrrC2 } & \multicolumn{2}{|l|}{ CG3 } & \multicolumn{2}{|c|}{ pX01-aat } & \multicolumn{2}{|c|}{ pXO2-at } \\
\hline & & & $S$ & $\bar{N}$ & $\mathrm{~S}$ & $\bar{N}$ & $\bar{S}$ & $\bar{N}$ & $\mathrm{~s}$ & $\bar{N}$ & $\bar{S}$ & $\bar{N}$ & $\mathrm{~s}$ & $\bar{N}$ & $\mathrm{~s}$ & $\mathrm{~N}$ & $\mathrm{~s}$ & $\mathrm{~N}$ \\
\hline GZBA01 & 2006001 & 2006 & 325 & 11 & 229 & 16 & 162 & 7 & 581 & 53 & 532 & 17 & 168 & 4 & 135 & 11 & 149 & 14 \\
\hline GZBA02 & 2006002 & 2006 & 325 & 11 & 229 & 16 & 162 & 7 & 581 & 53 & 514 & 16 & 168 & 4 & 132 & 10 & 149 & 14 \\
\hline GZBA03 & 2006003 & 2006 & 325 & 11 & 229 & 16 & 162 & 7 & 581 & 53 & 532 & 17 & 168 & 4 & 135 & 11 & 143 & 11 \\
\hline SZBA04 & 2006004 & 2006 & 325 & 11 & 229 & 16 & 162 & 7 & 581 & 53 & 532 & 17 & 168 & 4 & 135 & 11 & 145 & 12 \\
\hline GZBA05 & 2006005 & 2006 & 325 & 11 & 229 & 16 & 171 & 8 & 581 & 53 & 532 & 17 & 168 & 4 & 135 & 11 & 143 & 11 \\
\hline GZBA06 & 2006006 & 2006 & 325 & 11 & 229 & 16 & 189 & 10 & 581 & 53 & 532 & 17 & 168 & 4 & 138 & 12 & 143 & 11 \\
\hline GZBA07 & 2007001 & 2007 & 313 & 10 & 229 & 16 & 162 & 7 & 581 & 53 & 532 & 17 & 163 & 3 & 132 & 10 & 139 & 9 \\
\hline GZBA08 & 2007003 & 2007 & 313 & 10 & 229 & 16 & 189 & 10 & 581 & 53 & 532 & 17 & 163 & 3 & 132 & 10 & 145 & 12 \\
\hline ZBA09 & 2007004 & 2007 & 325 & 11 & 229 & 16 & 162 & 7 & 581 & 53 & 532 & 17 & 168 & 4 & 138 & 12 & 143 & 11 \\
\hline GZBA10 & 2007005 & 2007 & 325 & 10 & 229 & 16 & 162 & 7 & 581 & 53 & 532 & 17 & 163 & 3 & 147 & 15 & 143 & 11 \\
\hline GZBA11 & 2007007 & 2007 & 325 & 11 & 229 & 16 & 162 & 7 & 581 & 53 & 532 & 17 & 168 & 4 & 135 & 11 & 145 & 12 \\
\hline GZBA12 & 2007008 & 2007 & 349 & 13 & 229 & 16 & 162 & 7 & 581 & 53 & 532 & 17 & 158 & 2 & 135 & 11 & 143 & 11 \\
\hline GZBA13 & 2007013 & 2007 & 325 & 11 & 229 & 16 & 162 & 7 & 581 & 53 & 532 & 17 & 163 & 3 & 133 & 10 & 143 & 11 \\
\hline GZBA14 & 2007018 & 2007 & 313 & 10 & 229 & 16 & 162 & 7 & 581 & 53 & 532 & 17 & 158 & 2 & 138 & 12 & 143 & 11 \\
\hline GZBA15 & 2007019 & 2007 & 325 & 11 & 229 & 16 & 162 & 7 & 581 & 53 & 532 & 17 & 158 & 2 & 138 & 12 & 145 & 12 \\
\hline GZBA16 & 2007 & 2 & 313 & 10 & 238 & 17 & 162 & 7 & 1 & 53 & 532 & 17 & 163 & 3 & 132 & 10 & 43 & 11 \\
\hline GZBA17 & 2007023 & 2007 & 313 & 10 & 229 & 16 & 162 & 7 & 581 & 53 & 532 & 17 & 158 & 2 & 135 & 11 & 143 & 11 \\
\hline GZBA18 & 2007024 & 2007 & 313 & 10 & 229 & 16 & 162 & 7 & 581 & 53 & 532 & 17 & 158 & 2 & 138 & 12 & 149 & 14 \\
\hline GZBA19 & 2007029 & 2007 & 313 & 10 & 229 & 16 & 162 & 7 & 581 & 53 & 532 & 17 & 158 & 2 & 138 & 12 & 143 & 11 \\
\hline GZBA20 & 2008001 & 2008 & 313 & 10 & 229 & 16 & 162 & 7 & 581 & 53 & 532 & 17 & 163 & 3 & 132 & 10 & 143 & 11 \\
\hline GZBA21 & 2008002 & 2008 & 313 & 10 & 229 & 16 & 171 & 8 & 581 & 53 & 532 & 17 & 163 & 3 & 132 & 10 & 143 & 11 \\
\hline GZBA22 & 2009001 & 2009 & 313 & 10 & 238 & 17 & 162 & 7 & 581 & 53 & 532 & 17 & 158 & 2 & 135 & 11 & 143 & 11 \\
\hline GZBA23 & 2009002 & 2009 & 313 & 10 & 238 & 17 & 162 & 7 & 581 & 53 & 532 & 17 & 158 & & 138 & 12 & 143 & 11 \\
\hline GZBA24 & 2009003 & 2009 & 313 & 10 & 229 & 16 & 171 & 8 & 581 & 53 & 514 & 16 & 158 & 2 & 138 & 12 & 143 & 11 \\
\hline GZBA25 & 2009024 & 2009 & 325 & 11 & 229 & 16 & 162 & 7 & 581 & 53 & 532 & 17 & 158 & 2 & 132 & 10 & 145 & 12 \\
\hline GZBA26 & 2010001 & 2010 & 349 & 13 & 229 & 16 & 162 & 7 & 581 & 53 & 532 & 17 & 158 & 2 & 132 & 10 & 145 & 12 \\
\hline GZBA27 & 2010002 & 2010 & 325 & 11 & 229 & 16 & 162 & 7 & 581 & 53 & 543 & 17 & 158 & 2 & 132 & 10 & 143 & 11 \\
\hline GZBA28 & 2010007 & 2010 & 313 & 10 & 229 & 16 & 171 & 8 & 581 & 53 & 532 & 17 & 163 & 3 & 132 & 10 & 143 & 11 \\
\hline GZBA29 & 2010010 & 2010 & 325 & 11 & 229 & 16 & 162 & 7 & 581 & 53 & 532 & 17 & 158 & 2 & 132 & 10 & 145 & 12 \\
\hline GZBA30 & 2010011 & 2010 & 313 & 10 & 229 & 16 & 162 & 7 & 581 & 53 & 532 & 17 & 158 & 2 & 132 & 10 & 147 & 13 \\
\hline GZBA31 & 2011002 & 2011 & 313 & 10 & 229 & 16 & 162 & 7 & 581 & 53 & 532 & 17 & 158 & 2 & 135 & 11 & 147 & 13 \\
\hline GZBA32 & 2011003 & 2011 & 313 & 10 & 229 & 16 & 162 & 7 & 581 & 53 & 532 & 17 & 163 & 3 & 132 & 10 & 145 & 12 \\
\hline
\end{tabular}

S: fragment sizes (bp); N: copy numbers.

R1 - R30) of B. anthracis from other provinces in China and other countries.

\section{Genetic relationship based on clustering analysis}

MLVA data of 32 isolates from Guizhou province and 76 worldwide distributed isolates belonging to $30 \mathrm{MTs}$ (MT R1 to 30), which includes 19 isolates (9 MTs) from 11 other provinces of China (Hebei, Inner Mongolia, Qingdao, Changping, Jiangxi, Liaoning, Henan, Gansu, Sichuan,
Xinjiang, Hong-Kong) and 57 isolates (21 MTs) of 14 other countries (Australia, Canada, Germany, India, Indonesia, Japan, Namibia, South-Africa, Thailand, Turkey, UK, UNK, USA, and Zimbabwe) were used for comparison. The cluster tree based on isolates from Guizhou Province and worldwide distributed isolates showed they were divided into three clusters (Figure 1), designated A, B and C. All the 32 isolates were distributed in cluster A, which were further divided into A1, A2, A3 


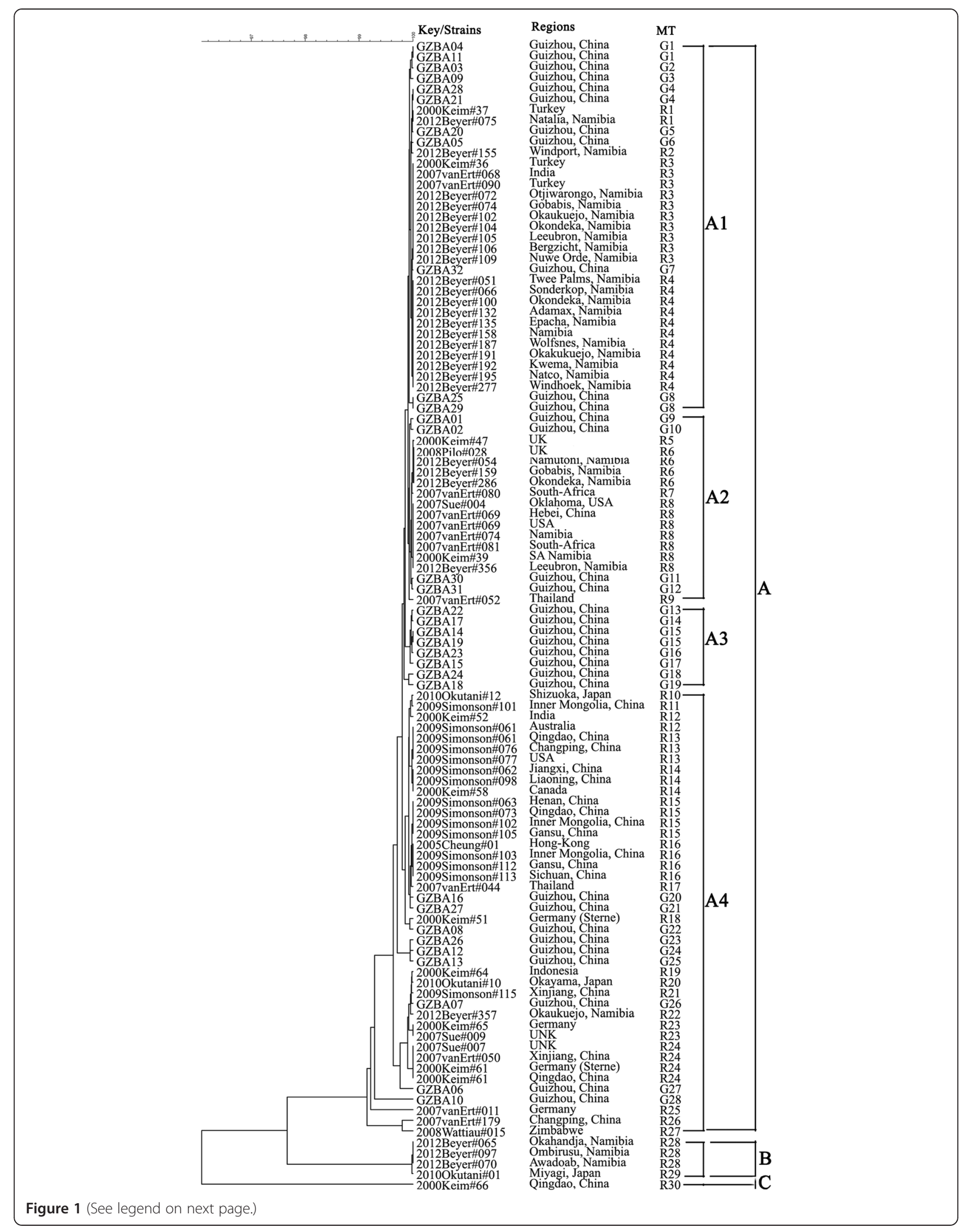


(See figure on previous page.)

Figure 1 Genetic relationships based on cluster tree of the 28 MLVA profiles of $B$. anthracis isolates from Guizhou province and isolates of worldwide distribution. The MLVA data of of isolates from other province of China and isolates of worldwide distribution were come from the B. anthracis data base (http://mlva.u-psud.fr/MLVAnet/spip.php?article123). The dendrogram based on the MLVA profiles of isolates from Guizhou Province and isolates of worldwide distribution were constructed using UPGMA.

and A4 sub-clusters. MT G1 - G8 isolates of Guizhou Province and MT R1-R4 isolates from India, Namibia and Turkey formed sub-cluster A1, in which isolates from Guizhou Province were relatively closely clustered. Sub-cluster A2 contains 18 isolates from Guizhou and Hubei Province of China, UK, South-Africa and Namibia. Isolates of MT G13-G19 from Guizhou Province formed the sub-cluster A3, while sub-cluster A4 includes 42 isolates (26 MTs) from China (including Guizhou, Inner Mongolia, Qingdao, Changping, Jiangxi, Liaoning, Henan, Gansu, Hongkong, Sichuan, Xinjiang Province) and other Countries, which included Asian countries such as Japan, Thailand and Indonesia and US, Germany, Zimbabwe, UNK and Namibia. It also showed that isolates from most of the Asian countries were grouped in sub-cluster A4, in which isolates from Guizhou were closely clustered. Four isolates from Zimbabwe, Namibia and Japan formed Cluster B, while isolates from Qingdao Province of China was separately formed the cluster $\mathrm{C}$.

\section{Genetic relationship based on minimum spanning tree}

Minimum Spanning Tree (MST) based on the MLVA data showed that the 28 MLVA profiles of isolates from Guizhou Province and 30 MLVA profiles of worldwide distributed isolates formed three clonal complexes (CCs) (G2, G8 and R16) and ten singletons (G10, G18, G21, R12, R18, R21, R25, R26, R27 and R30). All of isolates in CC G2 and CC G8 were come from Guizhou Province, while CC G14 contained isolates from both China (including Guizhou Province and other provinces of China) and other countries, in which isolates of MT G12, G14, G15, G18, G19 and G24 from Guizhou Province of China were relatively closely related, and the relationship of isolates of MT G4, G5, G7, G16, G20, G22, G25, G26 and G28 were relatively close in CC R16, which contained isolates of worldwide distribution (Figure 2).

\section{Discussion}

B. anthracis is a member of the Bacillus cereus group, containing Bacillus cereus, Bacillus thuringiensis, Bacillus mycoides and B. anthracis [16]. B. anthracis spores can remain stable for decades. This same longevity may greatly influence the ecology and evolution of this pathogen. The resting stage probably greatly reduces the rate of evolutionary change, and this may contribute to the extremely homogeneous nature of $B$. anthracis $[3,17]$.
Numerous studies have demonstrated the lack of molecular polymorphism within $B$. anthracis, which complicates efforts to subtype it [3,17-19]. The widely used molecular methods, such as Pulsed-field gel electrophoresis (PFGE) and multiple-locus sequence typing (MLST), often do not distinguish among closely related species, and many strains within a species show identical patterns (e.g., Bacillus cereus and B. anthracis) [3,20]. MLVA based on eight marker loci (MLVA-8) has been proved an effective molecular tool for discriminating among different $B$. anthracis isolates [3,15,21-23]. Therefore, we chose MLVA-8 to analyze the genetic characteristics of $B$. anthracis strains isolated in Guizhou Province and their relationship to strains of worldwide distribution. Anthrax is an important zoonosis in Guizhou Province in the history and it was first reported in Guizhou Province in 1957 [12,13]. Untill 1980s, the incidence in Guizhou Province exceeded the average in China and became the province with high incidence in 1990s, with incidence of $47.6 \%$ in 1991 [12]. Although the incidence reduced in recent year, sustained anthrax has been reported in recent years [13,14] and it was still more than the average level in China. At the same time, anthrax in animal is also a sever disease and caused sever economic lost in Guizhou Province [24]. The nationwide control system including disease outbreak surveillance has been carried out in Guizhou in the past few years and we recovered B. anthracis isolates from different epidemics. However, information on the genetic relationships of circulating B. anthracis isolates from Guizhou Province is lacking. Therefore, a total of 32 strains isolated from the local epidemics of cutaneous anthrax in Guizhou Province from 2006 to 2011 were used for analysis in this study. The results indicated that $32 \mathrm{~B}$. anthracis isolates were discriminated into $28 \mathrm{MTs}$, among which only four MTs (MT G1, G5, G13 and G19) were represent by more than one isolates, while the remaining MTs contained only one isolates. This suggested the genetic diversity of $B$. anthracis in Guizhou Province.

In order to analyse the relation of strains from Guizhou Province to strains of worldwide distribution, we compared each MLVA- 8 profiles of the 32 isolates in the $B$. anthracis data base (http://mlva.u-psud.fr/MLVAnet/spip. php?article123) to query identical MLVA profile in reported strains, and on identical profile was found in the online data base, which indicated that the 28 MLVA profiles of isolates from Guizhou Province were novel. Moreover, we downloaded MLVA-8 data of isolates from 


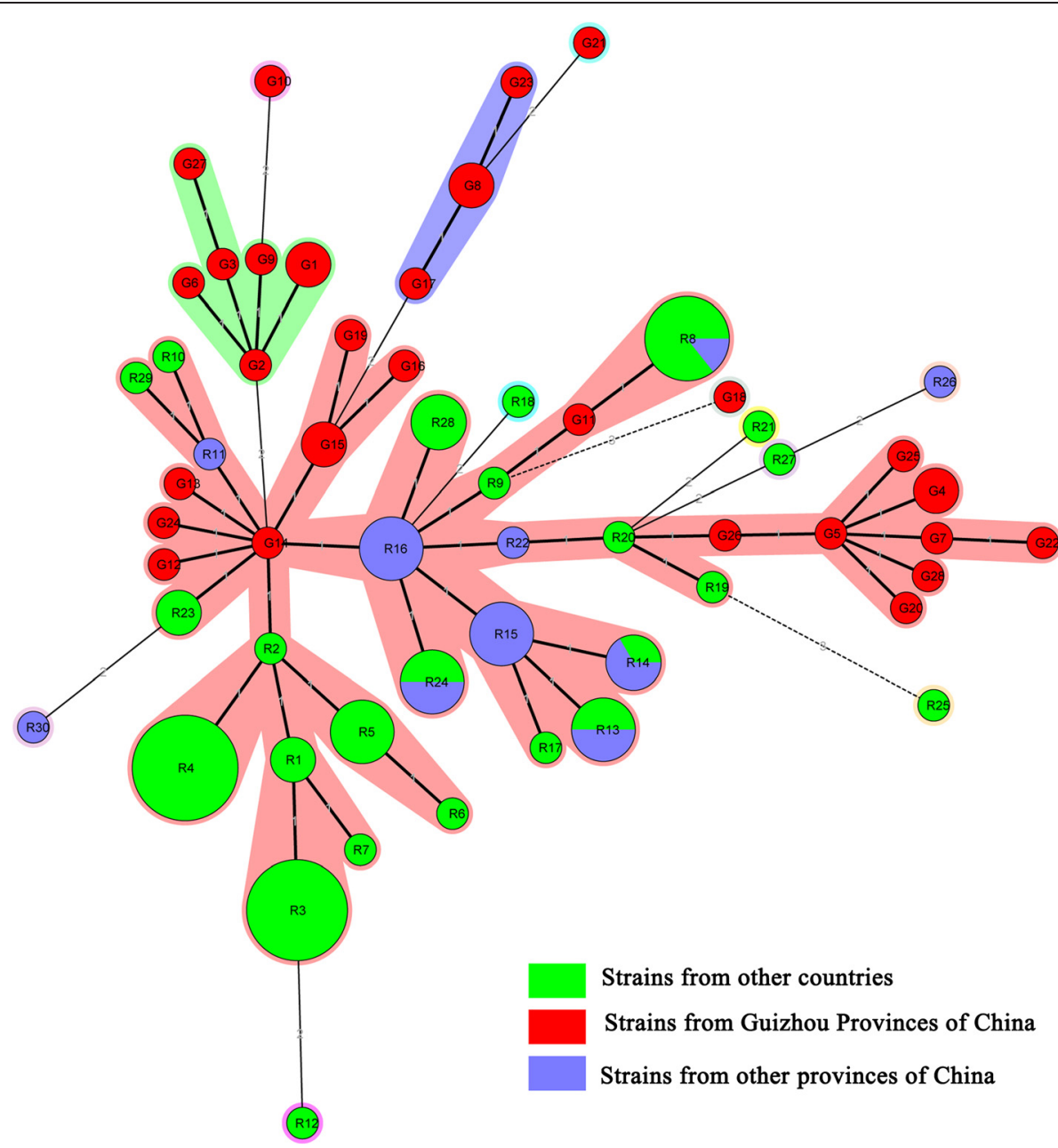

Figure 2 Genetic relationships based on minimum spanning tree of the 28 MLVA profiles of $B$. anthracis isolates from Guizhou province and isolates of worldwide distribution. Each circle corresponds to a MLVA profiles. The shadow zones in different colour correspond to different clonal complexes. The size of the circle is proportional to the number of the isolates, and the colour within the cycles represents the locality origin of Guizhou Province (red), other provinces of China (blue) or other countries (green).

other provinces of China and other countries from the $B$. anthracis data base for cluster analysis, a total of 76 worldwide distributed isolates belonging to 30 MTs inluding 19 isolates (9 MTs) from 11 other provinces of China and 57 isolates (21 MTs) of other countries were used for comparison. Cluster tree suggested that all the 32 isolates from Guizhou Province were distributed in cluster A (Figure 1) that can be further grouped into A1, A2, A3 and A4 subclusters, and most of isolates from Guizhou Province were relatively closely clustered in each sub-cluster, which suggested the relatively close relations of isolates from Guizhou Province. Besides, isolates of Chinese (including Guizhou and other provinces) and other Asian countries were grouped in sub-cluster A4, which suggested the relatively close relationship of isolates from Asian countries. Besides, MST tree clearly showed the relations of isolates of Guizhou Province and worldwide, in which two CCs (CC G2 and G8) contained isolates only from Guizhou Provinces, while CC R16 contained isolates from different countries, in which isolates from Guizhou Province were much more related. Our results will not only provide important information for prevention of anthrax and also enhances our understanding of genetic characteristics of B. anthracis in China.

\section{Conclusions}

A total of 32 isolates of $B$. anthracis from different anthrax epidemics in Guizhou Province during 2006 to 2011 were analysed using MLVA-8. 28 novel MTs of $B$. anthracis from Guizhou were revealed and the relationship to worldwide isolates was showed. The results will provide important information for prevention of anthrax and also enhances our understanding of genetic characteristics of B. anthracis in China. 


\section{Competing interests}

The authors declare that they have no competing interests.

\section{Authors' contributions}

$\mathrm{SL}$ and $\mathrm{HC}$ designed the study, analyzed the data and drafted the manuscript; $Q M$ and $Y L$ performed the MLVA experiments; $X W, L Y$, and KT participated in the isolation and identification of $B$. anthracis; GY, GT and DW participated in the epidemiological investigation and sample collection. All authors read and approved the final manuscript.

\section{Acknowledgements}

This work was supported by the grant of Guizhou Province Government Founds for Talent Base Construction for Infectious Disease Control and Prevention (No. Qian Ren Ling Fa [2013] 15) (Sub-project: Natural Focus and Insect-borne Infectious Disease Research Team [RCJD1401]). We acknowledge the help of Yan Wang from Chinese CDC for MLVA data analysis and the contribution of county CDC and prefecture CDC for sample collection.

\section{Author details}

${ }^{1}$ Institute of communicable disease control and prevention, Guizhou Provincial Centre for Disease Control and Prevention, 101 Bageyan Road, Guiyang 550004, Guizhou, People's Republic of China. 'uilyang Centre for Animal Disease Control and Prevention, Guiyang, China.

Received: 10 October 2014 Accepted: 12 March 2015

Published online: 31 March 2015

\section{References}

1. Hanna P. Anthrax pathogenesis and host response. Curr Top Microbiol Immunol. 1998;225:13-35.

2. Dixon TC, Meselson M, Guillemin J, Hanna PC. Anthrax. N Engl J Med. 1999;341(11):815-26.

3. Keim P, Price LB, Klevytska AM, Smith KL, Schupp JM, Okinaka R, et al. Multiple-locus variable-number tandem repeat analysis reveals genetic relationships within Bacillus anthracis. J Bacteriol. 2000;182(10):2928-36.

4. Beyer W, Turnbull PC. Anthrax in animals. Mol Aspects Med. 2009;30(6):481-9.

5. Zakowska D, Bartoszcze M, Niemcewicz M, Bielawska-Drozd A, Kocik J. New aspects of the infection mechanisms of Bacillus anthracis. Ann Agric Environ Med. 2012;19(4):613-8.

6. Khan AS, Morse S, Lillibridge S. Public-health preparedness for biological terrorism in the USA. Lancet. 2000;356(9236):1179-82.

7. Franz DR. Preparedness for an anthrax attack. Mol Aspects Med. 2009;30(6):503-10.

8. Simonson TS, Okinaka RT, Wang B, Easterday WR, Huynh L, U'Ren JM, et al. Bacillus anthracis in China and its relationship to worldwide lineages. BMC Microbiol. 2009;9:71.

9. Li CYS, Guo WD, Wang WR. Epidemiological analysis of anthrax in Inner Mongolia from 2001 to 2011. Chin J Zoonoses. 2012;28(8):854-6.

10. Ting-Lu Z, Liang-Liang C, Li L, Ming-Lei Z, Fang Q, Liang Y, et al. Investigation of an outbreak of cutaneous anthrax in Banlu village, Lianyungang, China, 2012. Western Pac Surveill Response J. 2012;3(4):12-5.

11. Luan YQ YC, Zhang ZX, Dong XQ. Epidemic situation of important naturalfocus diseases in Yunnan Province,1999-2008. Chin J Schisto Control. 2009;21(4):311-5.

12. TRPJaX Y. Analysis on epidemic chracteristic and reason of outbreak of anthrax in Guizhou Province. Chin J Epidemiol. 2000;22(5):393-4.

13. Zou ZTTR, He P. Analysis of epidemiological characteristics and causes of outbreaks of anthrax in Guizhou Province, 2000-2003. J med pest control. 2005;21(2):105-7.

14. Yao GH, Wang D, Guo J, Wei XY, Zeng Z, Tian KC, et al. [Response and evaluation of the disinfection effects on an anthrax outbreak inhuman being and cattle in Guizhou]. Zhonghua liu xing bing xue za zhi = Zhonghua liuxingbingxue zazhi. 2013;34(1):104-5.

15. Fasanella A, Van Ert M, Altamura SA, Garofolo G, Buonavoglia C, Leori G, et al. Molecular diversity of Bacillus anthracis in Italy. J Clin Microbiol. 2005;43(7):3398-401.

16. Turnbull PC. Definitive identification of Bacillus anthracis-a review. J Appl Microbiol. 1999:87(2):237-40.

17. Keim P, Kalif A, Schupp J, Hill K, Travis SE, Richmond K, et al. Molecular evolution and diversity in Bacillus anthracis as detected by amplified fragment length polymorphism markers. J Bacteriol. 1997;179(3):818-24.
18. Harrell LJ, Andersen GL, Wilson KH. Genetic variability of Bacillus anthracis and related species. J Clin Microbiol. 1995;33(7):1847-50.

19. Henderson I, Yu D, Turnbull PC. Differentiation of Bacillus anthracis and other 'Bacillus cereus group' bacteria using IS231-derived sequences. FEMS Microbiol Lett. 1995;128(2):113-8.

20. Lista F, Faggioni G, Valjevac S, Ciammaruconi A, Vaissaire J, Le Doujet C, et al. Genotyping of Bacillus anthracis strains based on automated capillary 25-loci multiple locus variable-number tandem repeats analysis. BMC Microbiol. 2006;6:33.

21. Hoffmaster AR, Fitzgerald CC, Ribot E, Mayer LW, Popovic T. Molecular subtyping of Bacillus anthracis and the 2001 bioterrorism-associated anthrax outbreak, United States. Emerg Infect Dis. 2002;8(10):1111-6.

22. Jung KH, Kim SH, Kim SK, Cho SY, Chai JC, Lee YS, et al. Genetic populations of Bacillus anthracis isolates from Korea. J Vet Sci. 2012;13(4):385-93.

23. Okutani A, Tungalag H, Boldbaatar B, Yamada A, Tserennorov D, Otgonchimeg I, et al. Molecular epidemiological study of Bacillus anthracis isolated in Mongolia by multiple-locus variable-number tandem-repeat analysis for 8 loci (MLVA-8). Jpn J Infect Dis. 2011;64(4):345-8.

24. Dapeng Yuan JY, Li Lliu Chunxiang Z, Hong J, Haixiang Y, Congbin Y, Dahu $Y$. Epidemic situation of livestock anthracnose and its control measures. Guizhou Agric Scincese. 2003;31(6):48-9.

\section{Submit your next manuscript to BioMed Central and take full advantage of:}

- Convenient online submission

- Thorough peer review

- No space constraints or color figure charges

- Immediate publication on acceptance

- Inclusion in PubMed, CAS, Scopus and Google Scholar

- Research which is freely available for redistribution 